\title{
Drug Accountability Dispensed Treatment Label Identifier Reference
}

National Cancer Institute

\section{Source}

National Cancer Institute. Drug Accountability Dispensed Treatment Label Identifier

Reference. NCl Thesaurus. Code C87870.

A character or string used to name or characterize the reference identifier for a dispensed treatment label with regards to a drug accountability assessment. 